\title{
Where do taxi trips start and end? Implications For Future Car-sharing For Commuting
}

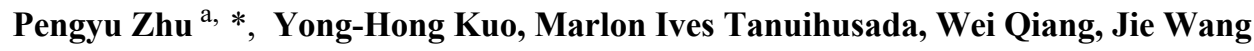 \\ a University of Hong Kong, brianzhu@hku.hk, \\ * Corresponding author
}

Keywords: taxi origination, built environment, car-sharing, commuting, peak hours

\section{Abstract:}

The impact of built environment on travel behaviour has long been an actively researched topic. However, many researches are focusing on the travel behaviour regarding to the choice of passengers on using public transport such as buses and subways, or private means such as private cars. Taxi, as a historical mean of public transport has not been widely researched in term of its relationship with the built environment.

Considering taxi as a practical, comfortable, and direct (door-to-door) means of transport, it is still widely used alongside with other available public transport modes either as a supplemental mode toward the buses and subways, or to accomplish the whole journey of a passenger. Of particular interest to transportation planners and policymakers is that a taxi trip which drives a passenger from a home to a bus stop or subway station, and vice versa might signifies its uses as either a first-mile trip or last-mile trip provider, supporting the inability of our public transit system to solve that problem in general. Therefore, the first research question of this paper is to investigate whether taxi has been able to increase urban accessibility via providing connection between homes and transit stations (i.e. subway stations or bus stops). Our model will control for a series of built environment features, especially the number of jobs within 100m of a transit station. Note that some commuters' taxi drop-off locations are indeed their workplace while others are simply the access points where they enter the public transit network (a.k.a. the first mile issue we mentioned). We use the variable 'number of jobs within 100m of a transit station' to rule out the blurring effect of the first scenario in which drop-off locations are workplaces. This is an important identification strategy for our first research question.

Aside from its function as a first-mile trip or last-mile trip provider and its relationship with the urban built environment, taxi has been commonly accepted as a mean of transport with significantly higher fare than either bus or subway, which is certainly an indicator of passengers' economic profile. In the meantime, racial minority groups tend to live in relatively separated areas, as referred in the residential segregation and spatial mismatch literature. These racial minority neighbourhoods, or racial and ethnic enclaves, often do not have good accessibility to public transit network. If we do find taxi is serving as a first-mile or last-mile trip provider, how is it related to the residential segregation of racial minority groups? Is it simply an affordability issue (i.e. determined by income)? Or are there something more that are inherent in the phenomenon of residential segregation? With this in mind, the second research question of this paper is to investigate whether taxi originations have been disproportionately low in racial minority neighbourhoods, after taking into account of income and a variety of other demographic and socioeconomic factors. If it is so, (relatively) affordable car sharing schemes provided by Uber, Lyft, and other subsidized vanpool programs might become very important for connecting these minority neighbourhoods to existing transit networks. The findings of this part would have important implications for equitable transportation planning and policymaking.

Combining big data analytics and traditional spatial statistical approach, the contributions of this paper are mainly two folds. First, it helps us understand whether taxi has been able to increase urban accessibility via providing connection between homes and transit stations (i.e. subway stations or bus stops). Second, it provides insights on whether taxi 
originations have been disproportionately low in racial minority neighbourhoods, raising transport equity concerns over a simple affordability issue.

This research aims to examine 1) whether taxi has been able to increase urban accessibility via providing connection between homes and transit stations (i.e. subway stations or bus stops); 2) whether taxi originations have been disproportionately low in racial minority neighbourhoods, raising transport equity concerns over a simple affordability issue. 